\title{
Changes in fatty acid composition of Neurospora crassa accompany sexual development and ascospore germination
}

\author{
Marta Goodrich-Tanrikulu, ${ }^{1}+$ Kelly Howe, ${ }^{2}$ Allan Stafford ${ }^{1}$ \\ and Mary Anne Nelson ${ }^{2}$
}

Author for correspondence: Mary Anne Nelson. Tel: +1 505277 2629. Fax: +1 5052770304.
e-mail: manelson@mail.unm.edu

1 United States Department of Agriculture, Agricultural Research Service, Western Regional Research Center, 800 Buchanan Street, Albany, CA 94710, USA

2 Department of Biology, University of New Mexico, Albuquerque, NM 87131, USA

\begin{abstract}
Fatty acid composition was determined during several stages of sexual development in Neurospora crassa. Triacylglycerol was the predominant acyl lipid in cultures undergoing sexual development. The absolute amounts of triacylglycerol in fertilized cultures varied over time, in contrast to control (unfertilized or mock-fertilized) cultures, in which the amount of triacylglycerol decreased linearly with age. In cultures competent to undergo sexual development, $\alpha$-linoleate was the predominant fatty acid, ranging from 53 to $65 \%$ of the total fatty acid mass. $\alpha$-Linolenate was $3 \%$ or less of the total fatty acid, in marked contrast to the much higher levels (10-35\%) typically reported for vegetative cultures. In fertilized cultures, a slightly higher mass ratio of oleate was also observed. This difference was due to the developing asci : in developing asci and mature ascospores, oleate replaced $\alpha$-linoleate as the predominant fatty acid (45 to $50 \%$ of the total). In germinating ascospores, the fatty acid composition approached that of vegetative cultures $6 \mathrm{~h}$ after inducing germination by heat activation. These results show that the fatty acid composition of sexual tissues of Neurospora differs substantially from the composition of asexual tissues, and that extensive changes in fatty acid composition correlate with several events in the sexual stage of development.
\end{abstract}

Keywords: fatty acids, filamentous fungi, lipids, Neurospora crassa, sexual development

\section{INTRODUCTION}

Neurospora crassa is a filamentous fungus that is able to undergo both asexual and sexual reproduction. Neurospora cultures become competent for sexual development when nitrogen is limiting, forming female sexual structures (protoperithecia). Fertilization of a protoperithecium by a strain of opposite mating type initiates formation of the perithecium (fruiting body) and leads to production of sexual progeny or ascospores (reviewed by Raju, 1992). In contrast to the much simpler structures of unicellular yeasts (in which isolated asci containing the sexual progeny develop in the absence of any supporting tissue), the fruiting body structures of filamentous fungi include both the sexual tissue and

†Present address: Calgene LLC, Monsanto, 1920 Fifth Street, Davis, CA 95616, USA.

Abbreviations: 16:0, palmitate; 16:1, palmitoleate; 18:0, stearate; 18:1, oleate; $18: 2, \alpha$-linoleate; $18: 3, \alpha$-linolenate. supporting asexual tissues. Sexual development in filamentous fungi thus requires sensing of appropriate environmental conditions, followed by interactions between the parental strains that ultimately lead to the production of several types of specialized tissue. Throughout development of the fruiting body, cell signalling occurs routinely (reviewed by Nelson, 1996). Over 200 mutations affecting sexual development in Neurospora have been identified (Raju, 1992). The defects of most mutants are not known.

Several diverse lines of evidence suggest that fatty acids or related lipids are important to sexual development of filamentous fungi. In Neurospora, unsaturated fatty acids, especially $\alpha$-linoleate $(18: 2)$, dramatically stimulate subsequent production of fruiting bodies when applied before fertilization (Nukina et al., 1981). We have observed even greater stimulation by applying oleate (18:1) (Nelson \& Oldrup, unpublished data), an unsaturated fatty acid not tested by Nukina $e t$ al. (1981). 
Similar observations have been made of the stimulation of oogonial development in Phytophthora cinnamomi (Zaki et al., 1983) and of the production of fruiting bodies in Nectria haematococca (Dyer et al., 1993) and Ceratocystis ulmi (Marshall et al., 1982) by unsaturated fatty acids. Furthermore, Neurospora ufa mutants, impaired in the desaturation of stearate $(18: 0)$ to $18: 1$, also have impaired fertility, which is improved by supplementation of crosses with 18:1 (Perkins \& Pollard, 1987; Goodrich-Tanrikulu et al., 1994). Mutants impaired in later fatty acid desaturation steps also have impaired fertility, which can be improved by adding unsaturated fatty acids to the crossing medium (Goodrich-Tanrikulu et al., 1995). In contrast, a mutant impaired in the synthesis of saturated fatty acids (cel) does not require fatty acid supplementation to yield fertile crosses. This evidence suggests a critical role for unsaturated fatty acids in sexual development.

Extracts of lipids from Neurospora cultures undergoing sexual development stimulate fruiting body formation (Islam, 1981). Extracts from Pyrenopeziza brassicae have a similar effect in many filamentous fungi, including Neurospora, and the active compounds appear to be structurally related to glycosphingolipids (Siddiq $e$ t al., 1989). Interestingly, disruption of a glycolipid transfer protein homologue in Podospora anserina also interferes with ascospore development (Saupe et al., 1994). In Aspergillus nidulans, fatty acid metabolites (hydroxylated 18:2 derivatives) known as psi factors have been shown to be inducers of sexual development (Mazur et al., 1990, 1991).

Almost no information on the fatty acid composition of sexual tissue of filamentous fungi is available. A variety of factors, such as culture age, the circadian rhythm, formation of macroconidia and microconidia, light and temperature, influence fatty acid composition of Neurospora, especially the relative levels of $18: 2$ and $\alpha$ linolenate (18:3) (Ballou \& Bianchi, 1978; Martin et al., 1981; Aaronson et al., 1982; Roeder et al., 1982; Vokt \& Brody, 1985; Coté \& Brody, 1987; Lyudnikova et al., 1990). However, fatty acid composition has been reported only for cultures undergoing asexual development. We anticipated that other environmental and developmental events might also influence fatty acid composition. This study provides information on the fatty acid and lipid composition of $N$. crassa during normal sexual development. We investigated the fatty acid composition of cultures competent for sexual development, as well as the composition of both sexual and asexual tissues at different stages during sexual development. Our study provides a foundation to examine signal transduction pathways specific to sexual development and to elucidate the defects of mutants in both sexual development and fatty acid metabolism.

\section{METHODS}

Strains. $N$. crassa strains were obtained from the Fungal Genetics Stock Center (FGSC), University of Kansas Medical Center, Kansas City, KS, USA. The following strains were used: the wild-type strains 74-OR23-IV $A$ (FGSC 2489) and ORS $a$ (FGSC 2490), and the fluffy strains $f l a$ (FGSC 4347) and $f A$ (FGSC 4317). $A$ and $a$ are the two mating types of $N$. crassa.

Cultures for fatty acid analysis. The $N$. crassa fluffy strain $(f l a)$ was used as female parent to minimize production of asexual spores (macroconidia) and to maximize production of perithecia (fruiting bodies). It was inoculated onto plates of Westergaard's crossing medium plus $1 \%(\mathrm{w} / \mathrm{v})$ sucrose (Westergaard \& Mitchell, 1947) covered with $82 \mathrm{~mm}$ circles of Miracloth (Calbiochem) to facilitate later removal of mycelia or perithecia for lipid analyses. After incubation for $7 \mathrm{~d}$ at $25^{\circ} \mathrm{C}$, the fluffy plates were either fertilized with $2 \mathrm{ml}$ of a $10^{8} \mathrm{ml}^{-1}$ conidial suspension from a wild-type strain of opposite mating type (74-OR23-IV $A$ ), mock-fertilized with the same number of conidia from a wild-type strain of the same mating type (ORS $a$ ), or left unfertilized. Incubations at $25^{\circ} \mathrm{C}$ in the light were continued for the times noted below.

At $0,2,5,7$ and $10 \mathrm{~d}$ post-fertilization (or after the mockfertilization), cultures were harvested by lifting off the Miracloth and adhering culture with forceps, cutting the Miracloth into four equal-sized sections and freezing each in liquid nitrogen. All samples were stored frozen until lipid extraction.

To test dependence of fatty acid composition on strain and culturing conditions, wild-type (74-OR23-IV $A$ ) or fluffy $(f l a)$ cultures were inoculated either onto Westergaard's crossing medium, or onto synthetic crossing medium or Vogel's medium N (Davis \& deSerres, 1970), covered with Miracloth as above and cultured at 25 or $34^{\circ} \mathrm{C}$ for 7 or $15 \mathrm{~d}$ before harvesting.

Isolation of asci (sexual tissue) and external fruiting body (asexual) tissue. The fluffy $(f l a)$ strain was inoculated onto crossing medium and incubated as described above. After $7 \mathrm{~d}$, the protoperithecia (female sexual structures) were fertilized by spreading the plates with suspensions of conidia of the wild-type strain 74-OR23-IV $A$. At 7, 10 and $13 \mathrm{~d}$ postfertilization, 100 perithecia were removed from the cultures and cleaned (external hyphal tissue removed). The asci within the fruiting bodies were extruded into a small amount of sterile water and immediately frozen in liquid nitrogen, after which all remaining tissue (the asexual external tissue) was separately frozen.

Germination of ascospores. Mature ascospores (500000) harvested from a cross of $f A \times$ ORS $a$ were added to $15 \mathrm{ml}$ tubes in $1 \mathrm{ml}$ Vogel's medium $\mathrm{N}$ with $1 \%$ sucrose. The suspensions of ascospores were heat-activated for $30 \mathrm{~min}$ at $65^{\circ} \mathrm{C}$ to break dormancy and incubated at $25^{\circ} \mathrm{C}$ with rocking to allow germination. For analyses, the ascospores or germlings were frozen in liquid nitrogen after the following treatments: no heat activation, immediately after heat activation $(0 \mathrm{~h})$, and at $3,6,12$ and $24 \mathrm{~h}$ after heat activation. Germination was about $50 \%$ at $6 \mathrm{~h}$ and reached approximately $95 \%$ by $24 \mathrm{~h}$.

Lipid extraction and fatty acid analyses. Frozen Miracloth sections and other samples were immersed in hot $\left(70^{\circ} \mathrm{C}\right)$ isopropyl alcohol to inactivate lipases, then extracted and analysed as described by Goodrich-Tanrikulu et al. (1994). In brief, aliquots of extracts were used to determine total fatty acid composition and aliquots were also applied to TLC plates to separate triacylglycerols from other neutral lipids and from polar lipids. Fatty acid methyl esters of triacylglycerols, polar lipids and total lipids were analysed by GLC, with methyl heptadecanoate as internal standard for quantification. 
Replication and statistical comparisons. As a test of variability, extracts from more than one of the four Miracloth sections of each culture were compared in preliminary studies. Variability among sampled sections was about $13 \%$. Remaining Miracloth sections were used for culture dry mass determinations, by subtracting the mass of equal-sized pieces of Miracloth alone.

Results for cultures sampled at each time after fertilization or germination were obtained from at least two experiments, generally in duplicate. Results from isolated asci and fruiting bodies of different ages were obtained from harvested material in three separate experiments.

We used InStat software (GraphPad Software) for statistical comparisons. The Student's $t$-test was used to assess the statistical significance of differences in values between treatment times, and between fertilized and unfertilized or mockfertilized cultures. Linear regression was used to evaluate the significance of changes over time.

\section{RESULTS AND DISCUSSION}

\section{Timing of sexual development}

Perithecia (fruiting bodies) are readily visible in Neurospora cultures within $2 \mathrm{~d}$ after fertilization, and by $5 \mathrm{~d}$ post-fertilization the fruiting bodies are filled with young asci. Within a fruiting body, development is asynchronous, so that at later stages both immature asci and those containing mature ascospores are present. Ascospore delineation is evident in many of the asci $7 \mathrm{~d}$ after fertilization. In the $10 \mathrm{~d}$ post-fertilization fruiting bodies, an entire range of asci is present, from those that have not formed even immature ascospores to those with mature (black) ascospores that will soon be ejected.
At $13 \mathrm{~d}$ post-fertilization, most of the asci have discharged their contents, but some asci contain spores (that will be ejected) and other asci lacking spores are still observed.

\section{Fatty acid and lipid composition in cultures competent for sexual development}

The lipid classes of Neurospora cultures competent for sexual development were the same as those observed in cultures at other developmental stages (Bianchi \& Turian, 1967; Ballou \& Bianchi, 1978; Aaronson et al., 1982; Goodrich-Tanrikulu et al., 1994). Specifically, fatty-acyl-containing lipids identified following TLC separation of extracts were phosphatidylcholine (the predominant polar lipid), phosphatidylethanolamine, phosphatidylinositol, triacylglycerols (the predominant neutral lipid) and diacylglycerols. Sterols and sterol esters were also present, as were smaller amounts of other lipids, including other phospholipids and unidentified compounds.

We used the superfertile fluffy $(f l)$ mutant of $N$. crassa (Lindegren et al., 1939) as the female parent to maximize the ratio of mating to vegetative tissues. Fluffy cultures grown under nitrogen limitation at $25^{\circ} \mathrm{C}$, conditions inducing formation of female sexual organs (protoperithecia), had much lower levels of $18: 3$ than usually reported for Neurospora (Table 1). The relative percentage $18: 3$ present in total lipids was $2-3 \%$ in cultures grown on plates of Westergaard's medium, compared to typical values of $10-35 \%$ in cultures grown at a similar temperature on plates of Vogel's medium $N$ (for

Table 1. Effect of strain and culture conditions on fatty acid composition of Neurospora cultures

Cultures were inoculated and grown for the time indicated as described in Methods, or, in the case of wild-type (wt) (macro)conidia, were harvested from cultures grown for $7 \mathrm{~d}$. The fluffy $(f)$ strain does not produce macroconidia. Neither fluffy nor wild-type produce protoperithecia on Vogel's medium N (VMN). On low-nitrogen media such as SCM (synthetic crossing medium) and Westergaard's, both fluffy and wild-type produce protoperithecia at $25^{\circ} \mathrm{C}$ but not at $34^{\circ} \mathrm{C}$. Relative percentage fatty acid composition of total lipids (mass basis) was determined after extraction and GLC of fatty acid methyl esters. Mean values from two experiments are shown. Variation in percentage $18: 3$ was less than $15 \%$.

\begin{tabular}{|c|c|c|c|c|c|c|c|c|c|}
\hline \multirow[t]{2}{*}{ Strain } & \multirow{2}{*}{$\begin{array}{c}\text { Temperature } \\
\left({ }^{\circ} \mathrm{C}\right)\end{array}$} & \multirow[t]{2}{*}{ Medium } & \multirow{2}{*}{$\begin{array}{l}\text { Time } \\
\text { (d) }\end{array}$} & \multicolumn{6}{|c|}{ Fatty acid } \\
\hline & & & & $16: 0$ & $16: 1$ & $18: 0$ & $18: 1$ & $18: 2$ & $18: 3$ \\
\hline wt & 25 & VMN & 7 & $15 \cdot 8$ & 0.5 & $3 \cdot 0$ & $15 \cdot 0$ & $59 \cdot 2$ & $6 \cdot 6$ \\
\hline wt & 25 & SCM & 7 & $18 \cdot 9$ & 0.4 & $6 \cdot 0$ & $14 \cdot 7$ & $56 \cdot 3$ & $3 \cdot 6$ \\
\hline wt & 25 & Westergaard's & 7 & $18 \cdot 9$ & $0 \cdot 4$ & $7 \cdot 6$ & $12 \cdot 3$ & $55 \cdot 1$ & $5 \cdot 8$ \\
\hline wt & 34 & Westergaard's & 7 & $22 \cdot 0$ & 0.6 & 3.7 & $11 \cdot 8$ & $54 \cdot 6$ & $7 \cdot 3$ \\
\hline $\begin{array}{l}\text { wt, } \\
\text { conidia }\end{array}$ & 25 & Westergaard's & 7 & $17 \cdot 2$ & $0 \cdot 9$ & $3 \cdot 5$ & $12 \cdot 7$ & $55 \cdot 3$ & $10 \cdot 3$ \\
\hline$f$ & 25 & VMN & 7 & $15 \cdot 6$ & 0.5 & $2 \cdot 8$ & $13 \cdot 7$ & $61 \cdot 8$ & $5 \cdot 6$ \\
\hline$f$ & 25 & SCM & 7 & $17 \cdot 5$ & 0.6 & $3 \cdot 6$ & $12 \cdot 0$ & $63 \cdot 8$ & $2 \cdot 6$ \\
\hline$f$ & 25 & Westergaard's & 7 & $18 \cdot 6$ & 0.6 & $4 \cdot 1$ & $12 \cdot 0$ & $61 \cdot 9$ & $2 \cdot 9$ \\
\hline$f$ & 34 & Westergaard's & 7 & $22 \cdot 1$ & 0.7 & $5 \cdot 0$ & $12 \cdot 5$ & $52 \cdot 9$ & $6 \cdot 1$ \\
\hline$f$ & 25 & Westergaard's & 15 & $16 \cdot 3$ & $1 \cdot 6$ & $2 \cdot 3$ & $11 \cdot 9$ & $66 \cdot 4$ & 1.5 \\
\hline
\end{tabular}


example, Vokt \& Brody, 1985; Coté \& Brody, 1987), a medium conducive to vigorous vegetative growth. Older cultures have a lower proportion of $18: 3$ (Vokt \& Brody, 1985). The low percentage of $18: 3$ observed in this study appears to be due to the cumulative effect of (1) advanced culture age at the time of fertilization; (2) slightly lower 18:3 content of fluffy cultures than of wild-type [probably because fluffy mutants do not produce macroconidia (Lindegren et al., 1939), which contain a high relative percentage of $18: 3]$; and (3) a lower proportion of $18: 3$ produced when Neurospora is grown on low-nitrogen media such as Westergaard's (Kushwaha et al., 1976) at temperatures conducive to sexual development. From comparison of fluffy and wild-type cultures grown under different conditions, we have confirmed that each of these factors is correlated with a slight reduction in the proportion of $18: 3$ produced (Table 1).

\section{Changes in lipids during sexual development}

We compared fertilized Neurospora cultures to two sets of control cultures, one consisting of unfertilized cultures and the other of mock-fertilized cultures ('fertilized' with a strain of the same mating type; no fruiting bodies develop under these conditions). This comparison allows identification of events specific to the fertilized cultures undergoing sexual development.

With the exception of triacylglycerol, the amounts of individual lipids visible by TLC did not change over time or among these treatments. However, the fatty acid content in polar lipids was analysed as a means of more precisely estimating quantitative changes in membrane lipids during sexual development. Polar lipid content fluctuated over time, but culture mass also fluctuated, averaging $160 \mathrm{mg}$ dry mass per plate. Consequently, the ratio of polar lipid fatty acid to culture mass was nearly constant for all treatments and times, averaging $5.5 \mu \mathrm{g}$ $\mathrm{mg}^{-1}$, showing a good correlation between membrane mass and culture mass.

The largest proportion of the total fatty acid in the cultures was derived from triacylglycerols, which contributed about $65 \%$ of the total fatty acid in unfertilized cultures, and from $40 \%$ (at $7 \mathrm{~d}$ ) to $65 \%$ of the total in both the fertilized and mock-fertilized cultures. At the time of fertilization, the mean triacylglycerol content was $27 \mu \mathrm{g}$ (mg dry mass) ${ }^{-1}$, or an absolute content of $4.2 \mathrm{mg}$ per plate. After this initial value, the triacylglycerol content of unfertilized cultures decreased linearly at a rate of $0.25 \mathrm{mg} \mathrm{d}^{-1}$; the triacylglycerol content of mock-fertilized cultures decreased faster, at a rate of $0.35 \mathrm{mg} \mathrm{d}^{-1}$ (Fig. 1). The difference between rates of decrease was significant (difference between slopes after linear regression significant at $95 \%$ confidence interval). The decrease in triacylglycerol content over time in these control cultures appears to be partly an effect of age of the culture, since it was observed in unfertilized cultures. The more rapid decrease in triacylglycerol content over time in mock-fertilized cultures than in unfertilized cultures may indicate a specific

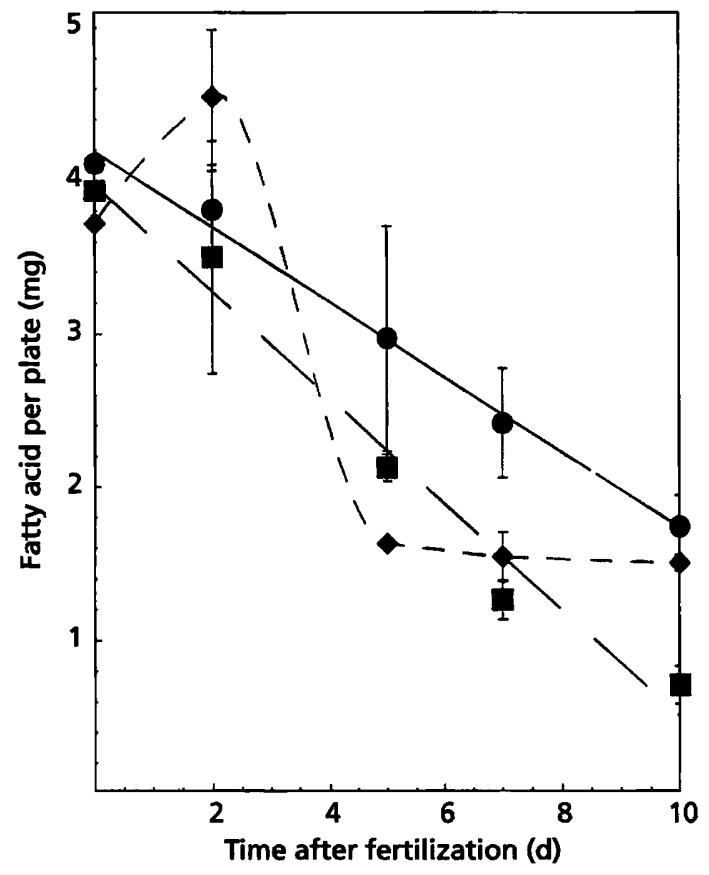

Fig. 1. Triacylglycerol content of crossed Neurospora cultures. Lipids were extracted from fluffy cultures either fertilized with wild-type conidia $(\triangleleft)$, left unfertilized $(0)$ or mock-fertilized with conidia of the same mating type $(\square)$. Triacylglycerols were separated from other lipids by TLC and quantified by GLC of fatty acid methyl esters. Data are means $\pm S E$ of two sets of duplicate cultures. Linear regression was used to obtain best-fit lines for unfertilized and mock-fertilized cultures; the slope of both lines differed significantly from 0 (no loss of triacylglycerol), and the difference between the standard error of the residuals for the two lines was also significant $(95 \%$ confidence interval), indicating that triacylglycerol content decreased at different rates for these cultures. Triacylglycerol content for the fertilized culture differs significantly from both unfertilized and mock-fertilized cultures at both 2 and $5 \mathrm{~d}$ (95\% confidence interval, Student's t-test).

response to the introduction of conidia not competent for fertilization.

In contrast, the triacylglycerol content of fertilized cultures was transiently higher, then lower relative to both control cultures (Fig. 1), and after $5 \mathrm{~d}$ remained constant. The transient increase in triacylglycerol content of fertilized cultures suggests rapid triacylglycerol synthesis within the first $2 \mathrm{~d}$ post-fertilization, correlating with perithecial enlargement. This increase was followed by re-utilization of about $65 \%$ of the stored triacylglycerol by $5 \mathrm{~d}$ post-fertilization. The accompanying increase in polar lipid content was much smaller, so that only a portion of the fatty acids lost from triacylglycerol $(11 \%)$ could have been converted into membrane lipids. The remainder presumably was metabolized via $\beta$-oxidation.

The mean total fatty acid for these same cultures was $42 \mu \mathrm{g}$ fatty acid in lipids per mg culture dry mass at the time of fertilization. For both the fertilized and mockfertilized cultures harvested just after fertilization, the 


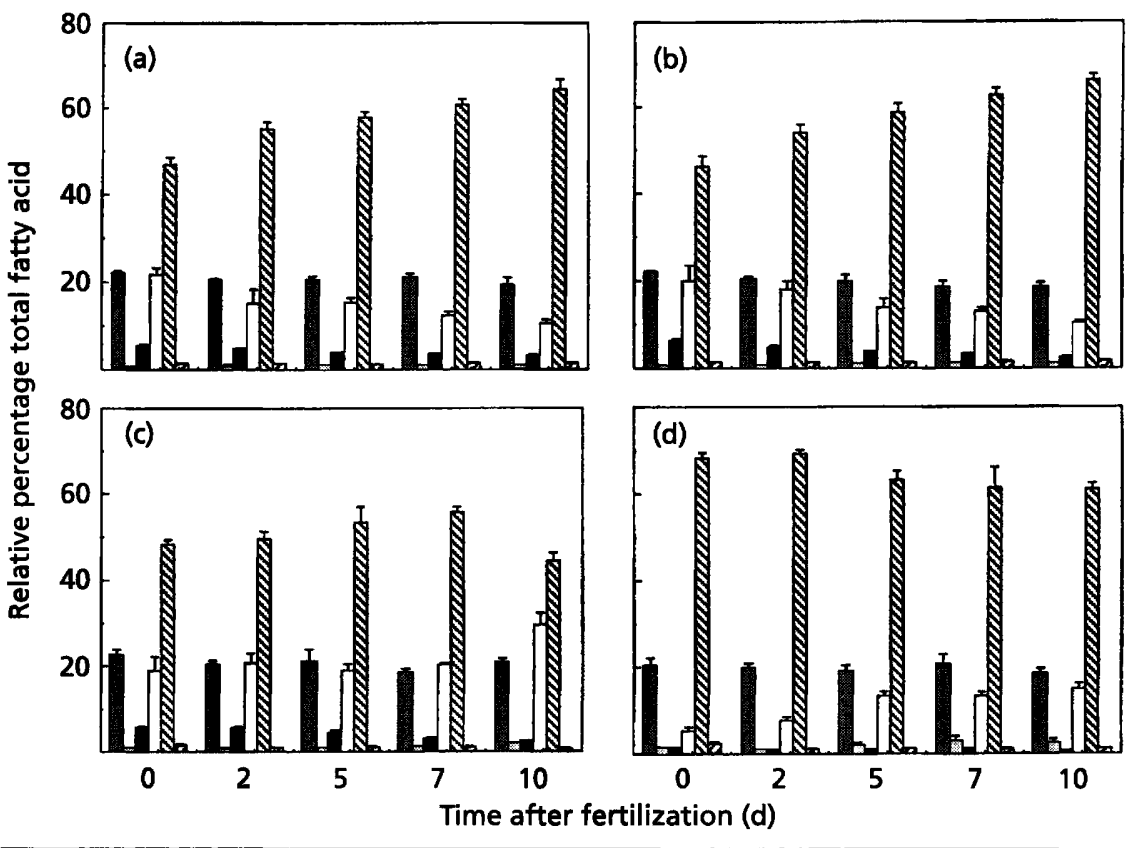

Fig. 2. Fatty acid composition of lipids in Neurospora cultures competent for sexual development. Triacylglycerol composition of (a) unfertilized, (b) mock-fertilized and (c) fertilized cultures. (d) Polar lipid (predominantly phosphatidylcholine) composition of fertilized cultures, undergoing sexual development. Lipids were extracted from fluffy cultures either fertilized with wildtype conidia, left unfertilized or mockfertilized with conidia of the same mating type. Triacylglycerols and polar lipids were separated from other lipids by TLC and quantified by GLC of fatty acid methyl esters. Data are means \pm SE of two sets of duplicate cultures. Triacylglycerol content was that in Fig. 1 and polar lipid content for (d) ranged between 900 and $1300 \mu \mathrm{g}$ per plate, depending on treatment time. 图,

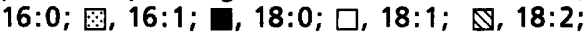
『18:3. fatty acid content and dry mass were slightly lower than for the controls left unfertilized. No contribution to the total fatty acids from the fertilizing conidia was observable.

Over time, the total fatty acid content of lipids from both unfertilized and mock-fertilized cultures reflected the decrease in triacylgycerol content. At $10 \mathrm{~d}$ postfertilization, the unfertilized cultures contained $23 \mu \mathrm{g}$ and the mock-fertilized $12 \mu \mathrm{g}$ total fatty acid (mg dry mass) $)^{-1}$. The fatty acid content of fertilized cultures over time showed more complex changes, increasing by $2 \mathrm{~d}$ post-fertilization to $50 \mu \mathrm{g}$ (mg dry mass) ${ }^{-1}$, decreasing rapidly by $5 \mathrm{~d}$ to $18 \mu \mathrm{g}$ (mg dry mass) ${ }^{-1}$ and then remaining almost constant through to $10 \mathrm{~d}$. The changes in fatty acid content of fertilized cultures were also due primarily to the changes in triacylglycerol content, rather than to changes in culture mass.

Total lipid fatty acid composition of the cultures reflected the relative contributions of triacylglycerols, polar lipids and other minor lipids, with 18:2 comprising between 53 and $65 \%$ of the total by mass for all treatments and times (not shown).

In addition to the observed decreases in amount of triacylglycerols, the fatty acid composition of triacylglycerols from both unfertilized and mock-fertilized cultures also changed over time. The most marked change was an increase of $18: 2$ from about $47 \%$ to $65 \%$ over $10 \mathrm{~d}$, with a corresponding decrease of 18:1 (Fig. $2 \mathrm{a}, \mathrm{b})$. In fertilized cultures, although the relative percentage of 18:2 in triacylglycerols initially increased, it subsequently decreased to $46 \%$ between 7 and $10 \mathrm{~d}$ post-fertilization, whereas the relative percentage of 18:1 increased during this same period; at $10 \mathrm{~d}, 18: 1$ made up $30 \%$ of the fatty acid in triacylglycerols of fertilized cultures (Fig. 2c), compared to $10 \%$ for both unfertilized and mock-fertilized cultures (Fig. 2a, b). An increase in relative percentage $18: 1$ of fertilized cultures was observed in polar lipids much sooner after fertilization (by $2 \mathrm{~d}$, prior to the delineation of asci; Fig. 2d). In contrast, the polar lipid fatty acid composition of both control cultures resembled that of fertilized cultures at time 0 and was unchanged over time (data not shown).

The considerably earlier increase in relative percentage 18:1 of fertilized cultures in polar lipids (Fig. 2d) compared to triacylglycerols (Fig. 2c) strongly suggests that the composition changes observed later in triacylglycerols are initiated in polar lipids. However, polar lipids have a higher relative percentage of $18: 2$ and lower percentage of 18:1 than do triacylglycerols (Fig. $2 c, d)$, suggesting that $18: 1$ is selectively incorporated into triacylglycerols, presumably in the developing ascospores or associated asexual tissues. The observed decrease in percentage 18:1 accompanying the decreases in triacylglycerol content (Fig. 1) in fertilized cultures between 2 and $5 \mathrm{~d}$, and in control cultures at all times examined, suggests that $18: 1$-containing triacylglycerols are being preferentially metabolized. 18:1 released from triacylglycerols by lipases may be $\beta$-oxidized, or desaturated to $18: 2$. Between 5 and $10 \mathrm{~d}$ post-fertilization, the amount of triacylglycerol was constant in the fertilized cultures (Fig. 1), so the changing triacylglycerol fatty acid composition between 7 and $10 \mathrm{~d}$ postfertilization indicates either turnover or retailoring of the composition of previously synthesized triacylglycerols as asci are maturing.

\section{Fatty acid composition of fruiting bodies and developing asci}

Fruiting bodies consist of sexual tissue (asci) and asexual tissue (external fruiting body tissue). To compare the fatty acid compositions of the sexual and asexual tissues, 


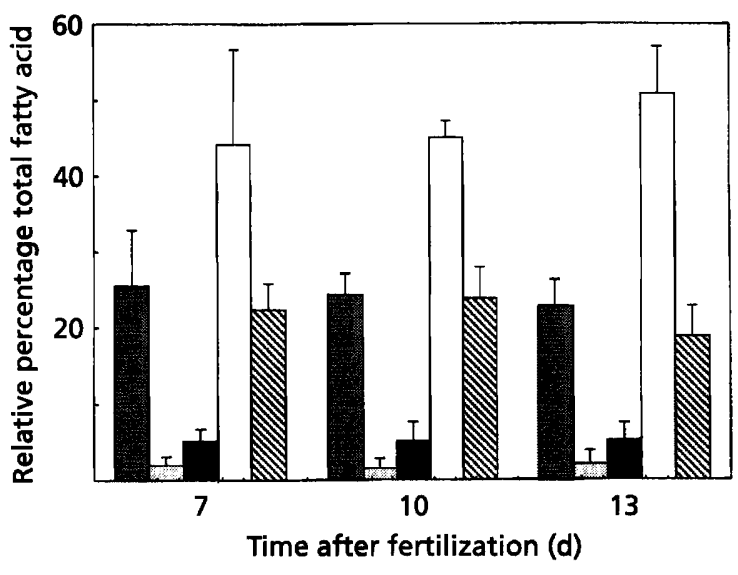

Fig. 3. Fatty acid composition of developing asci. Asci were isolated from fruiting bodies at 7, 10 and $13 \mathrm{~d}$ post-fertilization as described in Methods. Lipids in extracts were quantified by GLC of fatty acid methyl esters. Data are expressed as relative percentage fatty acid by mass (mean $\pm \mathrm{SE}$ ) and are from three independent isolations for each time point. Total fatty acid content was $0.32 \pm 0.05 \mu \mathrm{g}$ (mean \pm SE) for asci obtained from a single fruiting body. 圆, 16:0; $0,16: 1 ; \square, 18: 0 ; \square, 18: 1 ; \otimes$, 18:2.

we isolated fruiting bodies and separated the asci from the external tissue (at 7,10 and $13 \mathrm{~d}$ post-fertilization). Most of the fatty acid in both asci and the external tissue of fruiting bodies was present in the form of triacylglycerols (40-75\%, depending on developmental stage). Polar lipids typically accounted for another $9-12 \%$ of the total. Asci and ascospores also contained an unidentified lipid, not containing hydrolysable fatty acid. This compound may be sporopollenin, a carotenoidderived polymer reported to be present at high levels in ascospores (Brooks \& Shaw, 1978). Other lipids appeared to be absent. Mean fatty acid content for asci obtained from a single fruiting body was $0.3 \mu \mathrm{g}$ at each of the times after fertilization. Mean fatty acid content for emptied fruiting bodies was more variable among experiments $(0 \cdot 2-0.9 \mu \mathrm{g}$ per fruiting body) and did not appear to correlate with developmental age. Since the mass of fruiting bodies is considerably higher than that of asci, the percentage fatty acid content of asci is greater by mass.

The fatty acid composition of external (asexual) fruiting body tissue was similar to that of all Neurospora cultures (fertilized or control) grown on crossing medium at $25^{\circ} \mathrm{C}$, with high levels of $18: 2$ produced. Therefore, the high $18: 2$ content may be attributable partly to the presence of protoperithecia in the cultures. It can also be inferred that the fatty acid composition of protoperithecia is similar to that of the asexual perithecial tissues, although this was not directly tested, due in part to the difficulty of isolating substantial amounts of protoperithecia. A high 18:2 content appears to be a characteristic of cultures forming protoperithecia, and not simply due to growth on low-nitrogen medium, since fatty acid analyses of fluffy and wild-type cultures grown on Westergaard's medium at $25^{\circ} \mathrm{C}$ and at $34^{\circ} \mathrm{C}$ (a temperature at which protoperithecia do not form; Perkins, 1986) showed that the $34^{\circ} \mathrm{C}$-grown cultures had a lower proportion of $18: 2$ and a higher proportion of $18: 3$ (Table 1). This is the converse of the typical Neurospora temperature response, in which higher 18:3 is correlated with lower temperatures (see, for example, Martin et al., 1981; Vokt \& Brody, 1985).

In contrast to the asexual tissue of fruiting bodies, developing asci (as well as mature ascospores) contained an unusually high relative percentage of $18: 1$, typically ranging from 45 to $50 \%$ of the total fatty acids (Fig. 3). The increasing proportion of 18:1 in fertilized Neurospora cultures presumably reflects the contribution to total lipid from the increasing number of maturing asci present in the older cultures. The infertility of $u f a$ mutants when not provided with 18:1 (Perkins \& Pollard, 1987; Goodrich-Tanrikulu et al., 1994) may reflect an absolute requirement for a high proportion of $18: 1$ in developing asci. Consistent with this possibility, it has been observed that many (but not all) infertile Neurospora mutants have very low 18:1 in phospholipids, and furthermore, that all strains with low 18:1 are infertile (S. Brody \& V.E. A. Russo, personal communication).

\section{Changes in lipids during ascospore germination}

The fatty acid composition of ascospores before and immediately after heat activation to induce germination was indistinguishable, but over $24 \mathrm{~h}$, the relative percentage of 18:1 in polar lipids decreased (Fig. 4a, b). Following heat activation, total fatty acid in germinating ascospores cultured in Vogel's medium $\mathbf{N}$ increased linearly at a rate of $0.21 \mathrm{mg}$ per d per $10^{6}$ ascospores (not shown). In Neurospora tetrasperma, the primary source of metabolic energy in germinating ascospores is carbohydrate, although lipids can be utilized as a secondary source of energy, since in the absence of exogenously provided nutrients, total lipid content decreases by about half over 24 h (Lingappa \& Sussman, 1959).

Triacylglycerol accounted for much of the total fatty acid in germinating ascospores. Triacylglycerol content increased from about $60 \%$ of the total fatty acid in mature ascospores to $80-90 \%$ of the total between 3 and $12 \mathrm{~h}$ after heat activation. By $24 \mathrm{~h}$ after heat activation, triacylglycerol content had decreased to $40 \%$ of the total, with most of the remaining fatty acid (55\%) present in the form of polar lipids, suggesting utilization of triacylglycerols for the synthesis of membrane lipids. Fatty acid content in polar lipids increased most quickly during the same period, between 12 and $24 \mathrm{~h}$ after heat activation (from 32 to $140 \mu \mathrm{g}$ per $10^{6}$ ascospores). The amounts of three other lipid classes, tentatively identified as 1,2-diacylglycerols, free sterols and sterol esters, increased noticeably between 3 and $24 \mathrm{~h}$ after heat activation, as observed following TLC separations of total lipids (chromatogram not shown). The rapid production of membrane lipids such as phospholipids and sterols is expected, given the extensive mycelial growth which occurs during this time. 


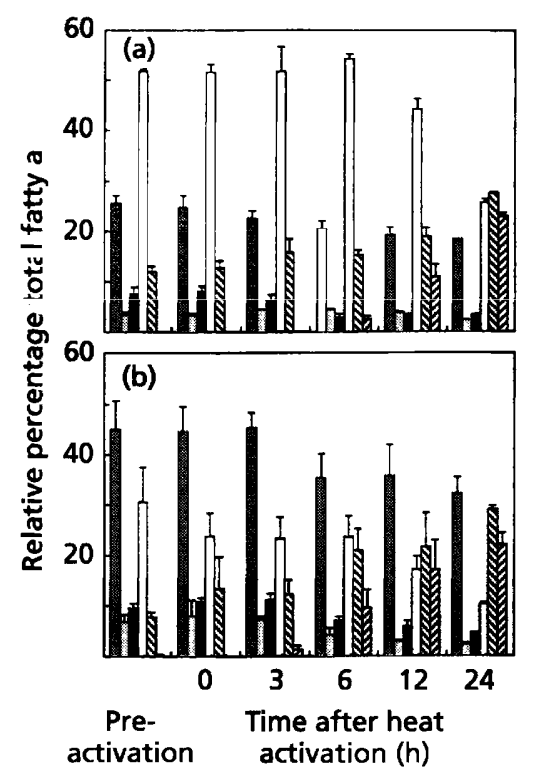

Fig. 4. Fatty acid composition of germinating ascospores. (a) Total lipid composition. (b) Polar lipid (predominantly phosphatidylcholine) composition. Lipids were extracted from ascospores before heat activation, and at the indicated times after heat activation followed by growth at $25^{\circ} \mathrm{C}$. Fatty acids were quantified by GLC of fatty acid methyl esters. Data are expressed as relative percentage fatty acid by mass (mean $\pm \mathrm{SE}$ ), and are from two sets of duplicate experiments. The mean total fatty acid content before heat activation was $44 \pm 8$ and polar lipid content $8 \pm 2 \mu \mathrm{g}$ per $10^{6}$ ascospores. After heat activation and growth for $24 \mathrm{~h}$, the respective values were $256 \pm 6$ and $70 \pm 8 \mu \mathrm{g}$ per $10^{6}$ ascospores. 图, 16:0; $\mathrm{0}, 16: 1 ; \square, 18: 0 ; \square$, $18: 1 ; \mathbb{Q}, 18: 2 ; \square, 18: 3$.

The fatty acid composition of germinating ascospores showed a gradual progression to the composition of typical vegetative cultures and was essentially typical by $24 \mathrm{~h}$ (Fig. 4a). In particular, the relative proportion of 18:1 decreased, and that of 18:2 and 18:3 increased.

The increasing proportion of $18: 3$ was noticeable earlier in polar lipids (Fig. 4b) than in total lipid (predominantly triacylglycerol; Fig. 4a). These observations, as well as observations of earlier changes in 18:1 composition in polar lipids compared to triacylglycerol (Fig. 2c, d), are consistent with a role of phosphatidylcholine as an intermediate in triacylglycerol biosynthesis. Desaturation of $18: 1$ to $18: 2$ and $18: 3$ occurs on a phosphatidylcholine substrate (Baker \& Lynen, 1971). During development and germination of ascospores, the activity of the $18: 1$ and $18: 2$ desaturases must be differentially regulated. During ascospore development, $18: 3$ is nearly absent and 18:1 accumulates, so the activity of the 18:1 desaturase must decrease and that of the $18: 2$ desaturase remain low. In contrast, during germination, the relative levels of both $18: 2$ and $18: 3$ increase, so the activity of both desaturases must increase.

Ascospores have most of their lipid in the form of triacylglycerols, with smaller amounts of phospholipids and only trace amounts of other lipids. In striking contrast, in macroconidia, $94 \%$ of the lipid is phospholipid (Bianchi \& Turian, 1967), and in microconidia, only trace amounts of triacylglycerol and phospholipid are present, the major lipids being sterols and sterol esters (Ballou \& Bianchi, 1978). Ascospores and macroconidia also differ dramatically in fatty acid composition. Macroconidia contain high relative levels of $18: 2$ and 18:3, and low levels of 18:1 (Table 1). Ascospores have only trace levels of $18: 3$ (pre-activation, Fig. 4a). A high proportion of $18: 3$ is strongly associated with cultures grown at low temperatures (Vokt \& Brody, 1985) and is believed to be partly responsible for adaptation to low temperatures. However, $18: 3$ is also highly susceptible to oxidation. Thus, the differing fatty acid composition of ascospores may at least partly account for several of their characteristic physiological features: inability to survive freezing temperatures, ability to survive heat needed for activation and longterm viability (Perkins, 1986).

\section{Conclusions}

With this study, fatty acid composition data are now available for $N$. crassa throughout its development. We have shown that the fatty acid composition of Neurospora cultures competent for sexual development differs substantially from the composition of cultures during asexual development. In addition, the fatty acid composition of developing asci is distinct from that of the asexual supporting tissue of the fruiting body. The lipid composition of ascospores is also distinct from both types of asexual spores formed by Neurospora. Both polar lipids and triacylglycerols undergo significant changes in amount or composition during several stages of sexual development, indicating that major changes in lipid metabolism correlate with specific developmental events.

\section{ACKNOWLEDGEMENTS}

We especially thank David Perkins. He made this study possible, through his interest and by initiating contact between M.A.N. and M.G.-T. We also thank Stuart Brody, Robert Metzenberg and Thomas McKeon for numerous invaluable discussions, feedback and insights related to this work, and Lisa Oldrup for technical assistance. This research was supported by US Public Health Service Grant GM47374 and a Human Frontier Science Program Organization Research Grant to M.A.N.

\section{REFERENCES}

Aaronson, L. R., Johnston, A. M. \& Martin, C. E. (1982). The effects of temperature acclimation on membrane sterols and phospholipids of Neurospora crassa. Biochim Biophys Acta 713, 456-462.

Baker, N. \& Lynen, F. (1971). Factors involved in fatty acyl CoA desaturation by fungal microsomes. The relative roles of acyl CoA and phospholipids as substrates. Eur J Biochem 19, 200-210.

Ballou, L. R. \& Bianchi, D. E. (1978). Lipids associated with microconidial differentiation in a mutant of Neurospora crassa. Curr Microbiol 1, 111-115. 
Bianchi, D. E. \& Turian, G. (1967). Lipid content of conidia of Neurospora crassa. Nature 214, 1344-1345.

Brooks, J. \& Shaw, G. (1978). Sporopollenin: a review of its chemistry, palaeochemistry and geochemistry. Grana 17, 91-97.

Coté, G. G. \& Brody, S. (1987). Circadian rhythms in Neurospora crassa: a clock mutant, prd-1, is altered in membrane fatty acid composition. Biochim Biophys Acta 904, 131-139.

Davis, R. H. \& deSerres, F. J. (1970). Genetic and microbiological research techniques for Neurospora crassa. Methods Enzymol 17A, 79-143.

Dyer, P. S., Ingram, D. S. \& Johnstone, K. (1993). Evidence for the involvement of linoleic acid and other endogenous lipid factors in perithecial development of Nectria haematococca mating population VI. Mycol Res 97, 485-496.

Goodrich-Tanrikulu, M., Stafford, A. E., Lin, J.-T., Makapugay, M. I., Fuller, G. \& McKeon, T. A. (1994). Fatty acid biosynthesis in novel $u f a$ mutants of Neurospora crassa. Microbiology 140 , 2683-2690.

Goodrich-Tanrikulu, M., Lin, J.-T., Stafford, A. E., Makapugay, M. I., McKeon, T. A. \& Fuller, G. (1995). Novel Neurospora crassa mutants with altered synthesis of polyunsaturated fatty acids. Microbiology 141, 2307-2314.

Islam, M. S. (1981). Sex pheromones in Neurospora crassa. In Sexual Interactions in Eukaryotic Microbes, pp. 131-154. Edited by D. H. O'Day \& P. A. Horgen. New York: Academic Press.

Kushwaha, S. C., Kates, M., Kramer, J. K. G. \& Subden, R. E. (1976). Lipid composition of Neurospora crassa. Lipids 11, 778-780.

Lindegren, C. C., Beanfield, V. \& Barber, R. (1939). Increasing the fertility of Neurospora by selective inbreeding. Bot Gaz 100, 592-599.

Lingappa, B. T. \& Sussman, A. S. (1959). Endogenous substrates of dormant, activated and germinating ascospores of Neurospora tetrasperma. Plant Physiol 34, 466-472.

Lyudnikova, T. A., Chernysheva, E. K., Bezzubov, A. A. \& Kritskii, M. S. (1990). Interrelationship of desaturation of fatty acids of phospholipids and the synthesis of carotenoids during adaptation of Neurospora crassa cells to temperature and light influence. Biochemistry (English translation of Biokbimiya) 55, 1647-1653.

Marshall, M. R., Hindal, D. F. \& MacDonald, W. L. (1982). Production of perithecia in culture by Ceratocystis ulmi. Mycologia 74, 376-381.

Martin, C. E., Siegel, D. \& Aaronson, L. R. (1981). Effects of temperature acclimation on Neurospora phospholipids: fatty acid desaturation appears to be a key element in modifying phospholipid fluid properties. Biochim Biophys Acta 665, 399-407.

Mazur, P., Meyers, H. V., Nakanishi, K., El-Zayat, A. A. E. \& Champe, S. P. (1990). Structural elucidation of sporogenic fatty acid metabolites from Aspergillus nidulans. Tetrabedron Lett 31, 3837-3840.

Mazur, P., Nakanishi, K., El-Zayat, A. A. E. \& Champe, S. P. (1991). Structure and synthesis of sporogenic psi factors from Aspergillus nidulans. J Chem Soc D Chem Commun 20, 1486-1487.

Nelson, M. A. (1996). Mating systems in ascomycetes: a romp in the sac. Trends Genet 12, 69-74.

Nukina, M., Sassa, T., Ikeda, M., Takahasi, K. \& Toyota, S. (1981). Linoleic acid enhances perithecial production in Neurospora crassa. Agric Biol Chem 45, 2371-2373.

Perkins, D. D. (1986). Hints and precautions for the care, feeding and breeding of Neurospora. Fungal Genet Newsl 33, 35-41.

Perkins, D. D. \& Pollard, V. C. (1987). Newly mapped chromosomal loci of Neurospora crassa. Fungal Genet Newsl 34, 52-53.

Raju, N. B. (1992). Genetic control of the sexual cycle in Neurospora. Mycol Res 96, 241-262.

Roeder, P. E., Sargent, M. L. \& Brody, S. (1982). Circadian rhythms in Neurospora crassa: oscillations in fatty acids. Biochemistry 21, 4909-4916.

Saupe, S., Descamps, C., Turcq, B. \& Bégueret, J. (1994). Inactivation of the Podospora anserina vegetative incompatibility locus $h e t-c$, whose product resembles a glycolipid transfer protein, drastically impairs ascospore production. Proc Natl Acad Sci USA 91, 5927-5931.

Siddiq, A. A., Ingram, D. S., Johnstone, K., Friend, J. \& Ashby, A. M. (1989). The control of asexual and sexual development by morphogens in fungal pathogens. Asp Appl Biol 23, 417-426.

Vokt, J. P. \& Brody, S. (1985). The kinetics of changes in the fatty acid composition of Neurospora crassa lipids after a temperature increase. Biochim Biophys Acta 835, 176-182.

Westergaard, M. \& Mitchell, H. K. (1947). Neurospora V. A synthetic medium favoring sexual reproduction. Am J Bot 34, 573-577.

Zaki, A. I., Zentmyer, G. A., Sims, J. J. \& Keen, N. T. (1983). Stimulation of sexual reproduction in the A2 mating type of Phytophthora cinnamomi by oleic acid and lipids from avocado roots. Phytopathology 73, 199-203.

Received 12 February 1998; accepted 26 March 1998. 